\title{
Non-targeted LC-MS based metabolomics analysis of the urinary steroidal profile
}

\author{
Amelia Palermo ${ }^{\text {a, b, c }}$, Francesco Botrè ${ }^{\text {c, d }}$, Xavier de la Torre ${ }^{\text {c }}$, Nicola Zamboni ${ }^{\text {a, * }}$ \\ a Institute of Molecular Systems Biology, ETH Zurich, Switzerland \\ ${ }^{\mathrm{b}}$ Department of Chemistry and Pharmaceutical Technologies, Sapienza University of Rome, Italy \\ ${ }^{\mathrm{c}}$ Laboratorio Antidoping, Federazione Medico Sportiva Italiana, Italy \\ ${ }^{\mathrm{d}}$ Department of Experimental Medicine, Sapienza University of Rome, Italy
}

\section{H I G H L I G H T S}

- A non-targeted LC-MS based workflow for the unbiased exploration of the urinary steroidal profile is presented.

- It detects up to 3000 metabolites including steroids of clinical and forensic relevance as glucurono- and sulfo-conjugates.

- The non-targeted exploration of urines after testosterone gel use reveals novel metabolites correlating with the intake.

\section{A R T I C L E I N F O}

\section{Article history:}

Received 27 October 2016

Received in revised form

23 January 2017

Accepted 24 January 2017

Available online 31 January 2017

\section{Keywords:}

Urinary steroidal profile

Non-targeted metabolomics

Anabolic androgenic steroids

Testosterone gel

Glucocorticoids

Liquid chromatography

Mass spectrometry
G R A P H I C A L A B S T R A C T

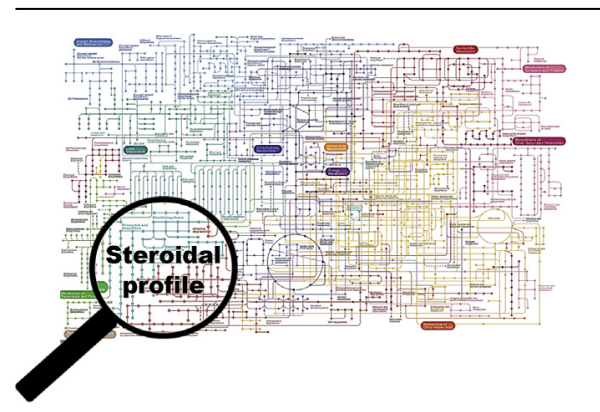

\begin{abstract}
A B S T R A C T
The urinary steroidal fraction has been extensively explored as non-invasive alternative to monitor pathological conditions as well as to unveil the illicit intake of pseudo-endogenous anabolic steroids in sport. However, the majority of previous approaches involved the a priori selection of potentially relevant target analytes. Here we describe the non-targeted analysis of the urinary steroidal profiles. The workflow includes minimal sample pretreatment and normalization according to the specific gravity of urine, a 20 min reverse phase ultra-performance liquid chromatographic separation hyphenated to electrospray time-of-flight mass spectrometry. As initial validation, we analyzed a set of quality control urines spiked with glucurono- and sulfo-conjugated steroids at physiological ranges. We then applied the method for the analysis of samples collected after single transdermal administration of testosterone in hypogonadal men. The method allowed profiling of approximately three thousand metabolic features, including steroids of clinical and forensic relevance. It successfully identified metabolic pathways mostly responsible for groups clustering even in the context of high inter-individual variability and allowed the detection of currently unknown metabolic features correlating with testosterone administration. These outcomes set the stage for future studies aimed at implementing currently monitored urinary steroidal markers both in clinical and forensic analysis.
\end{abstract}

() 2017 Elsevier B.V. All rights reserved.

\footnotetext{
* Corresponding author. Auguste-Piccard-Hof 1, 8093 Zurich, Switzerland

E-mail address: nzamboni@ethz.ch (N. Zamboni).
}

\section{Introduction}

In the last decades the urinary steroidal profile has caught increasing interest as an alternative, non-invasive way to monitor 
the insurgence of pathological conditions and the effectiveness of related therapies [1-8]. Its diagnostic value has been explored in adrenal tumors as well as prostate cancer, hypogonadism, and other secondary sexual dysfunctions [9-12]. In anti-doping tests, it has been considered for the detection of systemic intake of cortisone and hydrocortisone [13] and currently provides the reference landscape to unveil the use of endogenous anabolic steroids [14-16]. In this regard, testosterone and its precursors are one of the most abused classes of doping substances in sport. Although the unambiguous, direct confirmation of the exogenous origin of these steroids is obtained by gas chromatography coupled to isotopic ratio mass spectrometry (GC-IRMS) [17-19], this technique is still relatively complex and costly, and it is not yet performed on all urine samples received by the anti-doping laboratories. Consequently, the initial testing procedure relies on the detection and quantification of targeted steroids in urine. More precisely, since 2014 the World Anti-Doping Agency has approved the so called "Steroidal Module" of the Athlete Biological Passport (ABP), meant as a record over time for each athlete of the urinary concentrations of six steroidal markers (Testosterone (T), Epitestosterone (E), Androsterone (Andro), Etiocholanolone (Etio), 5 $\alpha$-androstane$3 \alpha, 17 \beta$-diol ( $5 \alpha$ Adiol) and $5 \beta$-androstane- $3 \alpha, 17 \beta$-diol (5 $\beta$ Adiol)) as sum of free and glucuronides $(G)$, together with their relative ratios and the specific gravity of the sample [20-22]. Obtained data are integrated in a Bayesian adaptive model that allows for the progressive replacement of previously applied population-based thresholds with personalized reference ranges [23]. The recent introduction of this strategy in routine analysis led to a significant improvement of the efficacy of the initial testing procedure for pseudo-endogenous anabolic androgenic steroids. Indeed, high level of specificity and robustness has been demonstrated in the case of oral or parenteral intake of T and related steroids [24-27]. However, its limits, for instance in the recognition of topical applications and in the context of long-term regimes, have been recently highlighted and still constitute an open question [28-32].

These aspects raised the need of developing alternative methodologies allowing the comprehensive exploration of the urinary steroidal fraction in the perspective of an evolution of the steroidal module towards a more resolved overview of metabolic modifications indicative of the doping offence. In this regard, according to classical targeted approaches, the general strategy followed for the development and implementation of analytical methods aimed at unraveling the intake of exogenous compounds includes the evaluation of the metabolic stability and of the excretion profile of the substance in order to select the most appropriate analytical marker(s) [33] (Fig. 1). This task is usually achieved performing in vitro and/or in vivo metabolism studies in combination with gas or liquid chromatography - mass spectrometry detection. The formation of specific metabolites is hypothesized on the basis of the current available knowledge and confirmed by the interpretation of data obtained by targeted analysis. Unfortunately, a priori assumptions often lead to results strongly biased by the absence of a complete understanding of the metabolic scenario in the biological system under investigation. In addition, even if not always predictable during the study design phase, significant quantitative and qualitative differences of the excretion profile have been observed depending on the particular route of administration of the substance, reflecting diverse sequences of metabolic reactions and enzymes distribution [30,34-36].

In an effort to encompass these matters and to increase the metabolic coverage of available methodologies, system-level approaches have recently found a variety of applications in biomarkers discovery. In particular, early studies in this direction have already unearthed previously unsuspected metabolic connections also in the anti-doping field. This is for example the case reported in a recent work by Wang et al. describing the association between salbutamol administration and purine metabolism [37]. Kiss et al. applied a non-targeted strategy to explore metabolic differences in urines collected by a group of volunteers and sportsmen and the changes induced by the administration of salbutamol or budesonide, remarking the utility of global approaches in the study of large cohorts of samples presenting high inter-individual variability [38]. Concerning endogenous anabolic androgenic steroids, the use of $\mathrm{T}$ formulations by oral route has been tackled by Boccard et al. pointing out the importance of detecting both glucuronated and sulfated (S) steroidal conjugates in urine [39]' and suggesting a set of biomarker candidates [40]. In a further investigation, Raro et al. revealed the increase in 1-cyclopentenoylglycine excretion in urine after intramuscular injection of T cypiotane [41]. These findings have extended the currently available information in support of a scrupulous examination of data acquired in routine analysis, endorsing the utility of comprehensive, fast and reliable analytical workflows for the broad exploration of the metabolic content of samples in a non-targeted fashion [42].

Here we present a comparative metabolomics strategy based on ultra-performance liquid chromatography - time of flight mass spectrometry with electrospray ionization (UHPLC-ESI-qTOF MS) and data processing in the open source OpenMS [43] environment for the non-targeted exploration of the urinary metabolic profile, ensuring the detection of steroids of relevance in clinical and antidoping research directly in the form of $G$ and $S$ conjugate metabolites (Fig. 2). In order to validate the approach, groups of urine samples spiked with endogenous steroid metabolites at concentrations within human physiological ranges were analyzed. We eventually applied the non-targeted strategy to explore the metabolic signature of samples collected before and after single transdermal administration of testosterone gel in a population of hypogonadal non-hospitalized patients.

\section{Materials and methods}

\subsection{Chemicals and reagents}

Solvents and chemicals were of analytical or HPLC grade and provided by Sigma-Aldrich (Schnelldorf, Switzerland). Water was obtained from a MilliQ water purification system (Millipore S.p.A., Milano, Italy). API-TOF reference mass solution kit containing purine, ammonium trifluoroacetate (ammonium TFA) and hexakis $(1 \mathrm{H}, 1 \mathrm{H}, 3 \mathrm{H}$-tetrafluoropropoxy) phosphazine (HP-0921) was from Sigma-Aldrich (Schnelldorf, Switzerland). Internal deuterated standards (d4-androsterone glucuronic acid (AndroG$\mathrm{d} 4), \mathrm{d} 4$-androsterone sulfate (AndroS-d4) triethylammonium salt, $\mathrm{d} 4-5 \alpha$-androstan-3 $\alpha, 17 \beta$-diol-17 $\beta$-glucuronic acid (5 $\alpha$ AdiolG-d4), d3-5 $\beta$-androstan-3 $\alpha, 17 \beta$-diol-17 $\beta$-glucuronic acid (5 3 AdiolG-d3)) and other steroidal standards (epitestosterone glucuronic acid (EG), testosterone glucuronic acid (TG), etiocholanolone glucuronic acid (EtioG) sodium salt, androsterone sulfate (AndroS) triethylammonium salt, dehydroisoandrosterone sulfate (DHEAS) sodium salt dehydrate) were from LGC Standards (Milano, Italy). Stock standard solutions were prepared in methanol at the concentration of $1 \mathrm{mg} /$ $\mathrm{mL}$ and $0.01 \mathrm{mg} / \mathrm{mL}$ (referred to the salt) and stored at $-20^{\circ} \mathrm{C}$ until use.

\subsection{Sample preparation}

Urine samples were thawed at room temperature and subject to a minimal sample preparation protocol including volume normalization according to the specific gravity (SG) of the sampleBriefly, SG was measured by refractometry and variable aliquots of samples, calculated with the formula $100 \mu \mathrm{L}^{*} 1.02 / \mathrm{SG}$, were transferred 


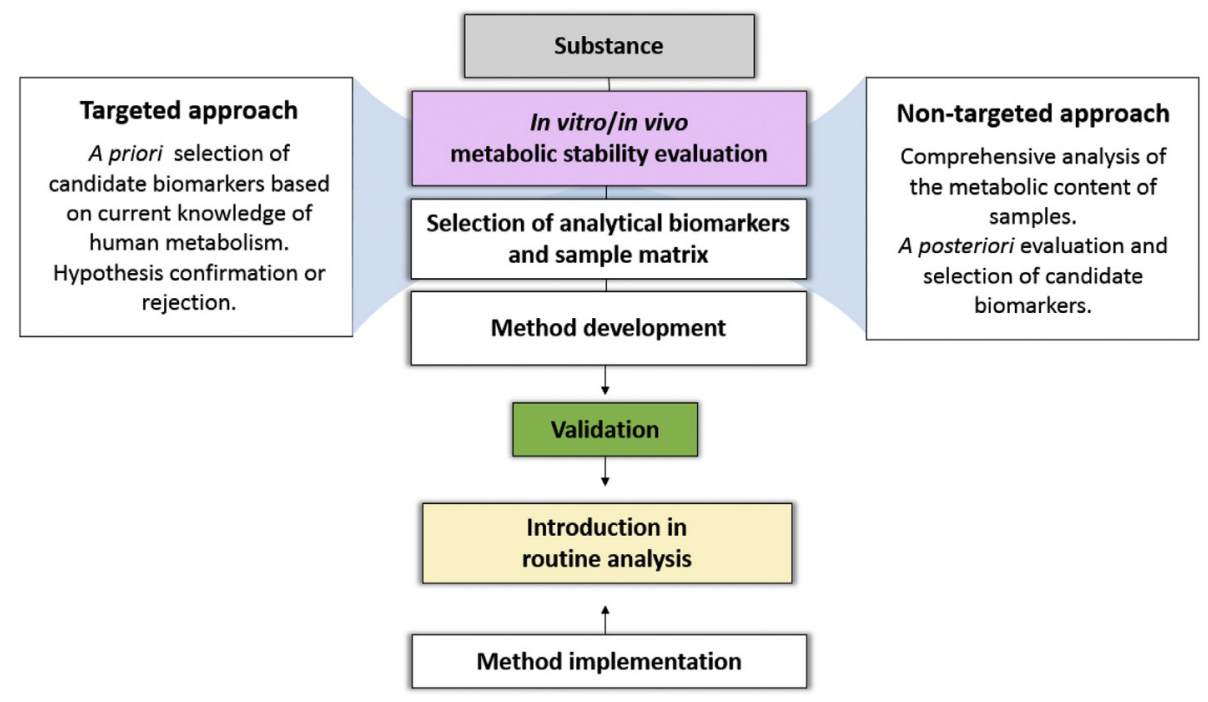

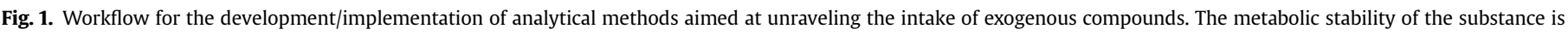
assessed either in vitro or in vivo performing classical targeted hypothesis testing studies or with more comprehensive non-targeted approaches.

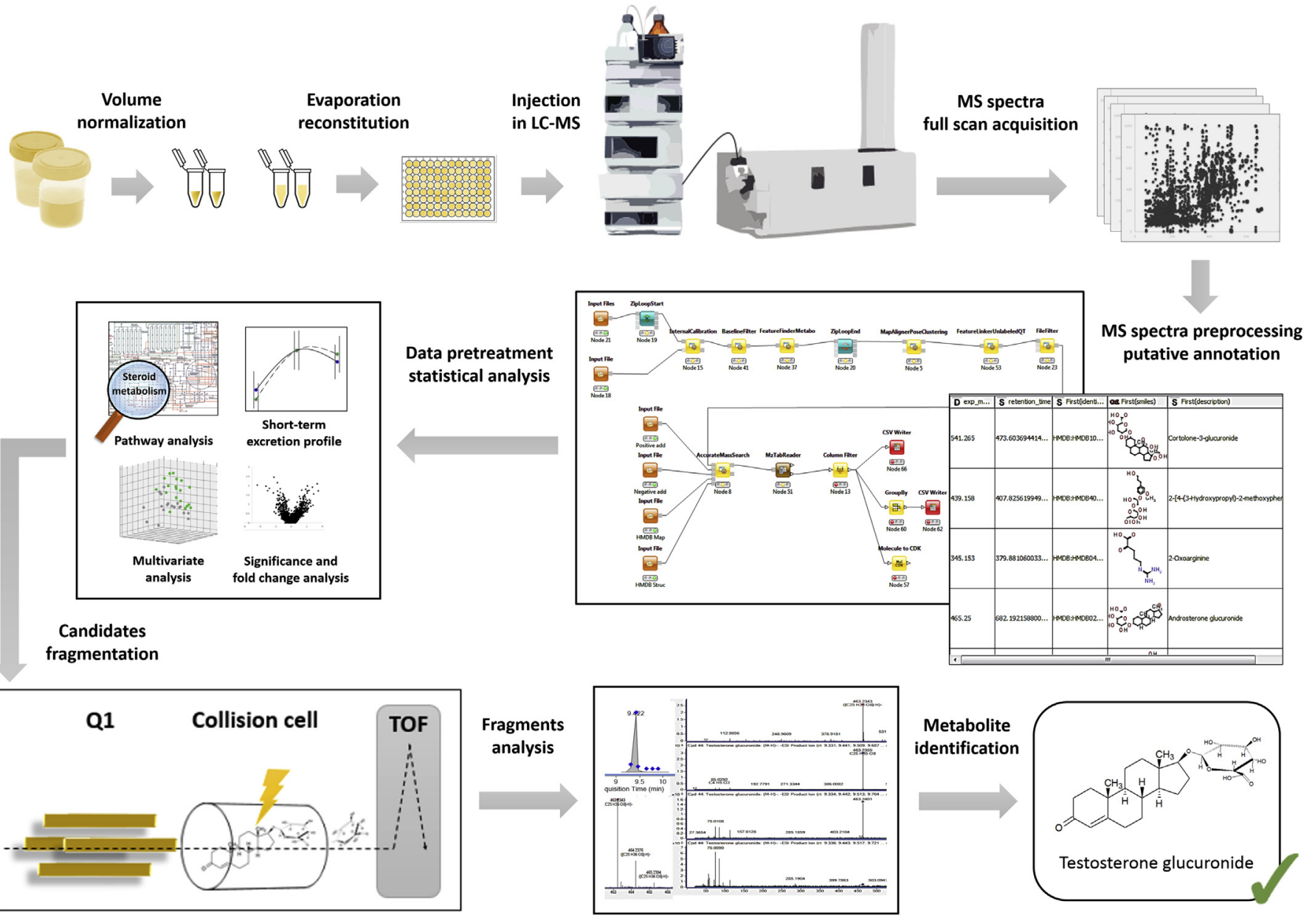

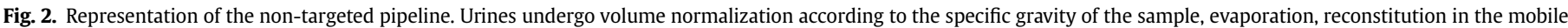

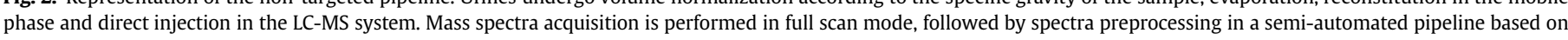

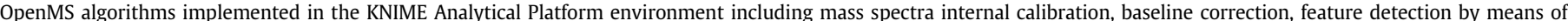

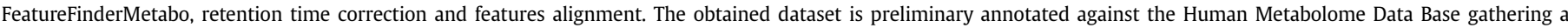

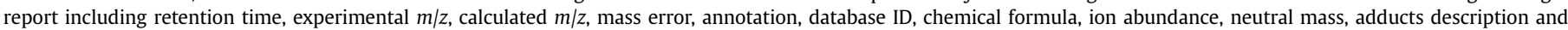

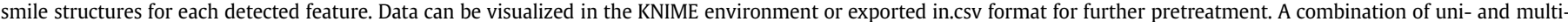

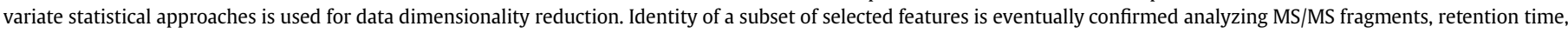
isotopic pattern and standards. 
in $1.5 \mathrm{~mL}$ Eppendorf tubes. $10 \mu \mathrm{L}$ of a methanolic solution containing four deuterated standards (AndroG-d4, AndroS-d4,

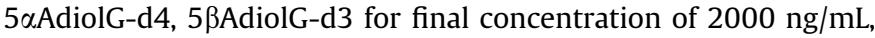
$2000 \mathrm{ng} / \mathrm{mL}, 100 \mathrm{ng} / \mathrm{mL}$ and $100 \mathrm{ng} / \mathrm{mL}$, respectively) were added and samples evaporated to dryness under vacuum at room temperature. For spiked quality control samples, additional $10 \mu \mathrm{L}$ of a methanolic solution containing EG, TG, EtioG, AndroS and DHEAS (for final concentrations of 100,100, 2000, 2000, and $2000 \mathrm{ng} / \mathrm{mL}$, respectively) were added.

After evaporation, all samples were reconstituted in $100 \mu \mathrm{L}$ of acetonitrile/weather $(10 / 90 \mathrm{v} / \mathrm{v})$, vortexed, centrifuged at $10.000 \mathrm{~g}$ at $4{ }^{\circ} \mathrm{C}$ for $5 \mathrm{~min}$, the supernatant transferred in a 96-well plate and injected in the UHPLC-qTOF system. In order to increase the sensitivity for steroidal metabolites in physiological concentration ranges, different sample dilutions were evaluated (including $10,5,2$ fold), undiluted and 5, 10-fold concentration as well as different compositions of the reconstitution solution.

\subsection{Chromatographic and mass spectrometric conditions}

Chromatographic separation was performed using an ultra-high pressure chromatographic system (UHPLC) equipped with an Acquity UHPLC BEH C18 $(2.1 \times 5 \mathrm{~mm}, 1.71 .7 \mu \mathrm{m})$ Van Guard precolumn in combination with an Acquity UHPLC BEH C18 column $(2.1 \times 150 \mathrm{~mm}, 1.7 \mu \mathrm{m})$. As mobile phases $0.01 \%$ formic acid in water (A) and $0.01 \%$ formic acid in acetonitrile (B) were used. After evaluation of different elution gradients and column temperatures, the final conditions were as follow: $15 \%$ B from 0 to $1 \mathrm{~min}, 40 \% \mathrm{~B}$ from 1 to $8.2 \mathrm{~min}, 40 \% \mathrm{~B}$ until $9.8 \mathrm{~min}, 95 \% \mathrm{~B}$ from 9.8 to $13.5 \mathrm{~min}$, 95\% B until 14.5 min, 15\% B from 14.5 until the end of the run. The total chromatographic run time was $16.5 \mathrm{~min}$, column temperature $30^{\circ} \mathrm{C}$ and flow rate at $0.3 \mathrm{~mL} / \mathrm{min}$. Samples were maintained at $8{ }^{\circ} \mathrm{C}$ until injection of $10 \mu \mathrm{L}$ in the UHPLC/MS system.

Liquid chromatographic mass spectrometric profiles were acquired on an Agilent 6520 Quadrupole Time of Flight (Agilent Technologies) with an electrospray ion source operated in dual ESI negative mode. Gas temperature was set at $365^{\circ} \mathrm{C}$, drying gas at $6 \mathrm{l} /$ min and nebulizer pressure at $30 \mathrm{psig}$. The capillary voltage was at $3500 \mathrm{~V}$, fragmentor at $200 \mathrm{~V}$ and skimmer at $65 \mathrm{~V}$. Mass acquisition was performed at $1 \mathrm{spectra} / \mathrm{s}$ for a total transients/spectrum of 9902. For continuous on line recalibration of $m / z$ axes TFA anion $(\mathrm{m} /$ $z$ 112.985587) and HP-0921 ( $m / z$ 1033.988109) were infused in the MS system with lock spry nebulizer pressure set at 10 psig. Full scan centroided mass spectra were acquired in $4 \mathrm{GHz}$ high resolution mode in the range $50-1050 \mathrm{~m} / \mathrm{z}$. For tandem MS fragmentation studies, the quadrupole was set to allow the selection of targeted $[\mathrm{M}-\mathrm{H}]$ ions in narrow $(1.3 \mathrm{~m} / \mathrm{z})$ isolation width mode. Precursor ions were fragmented at fixed collision energies (10, 20 and $40 \mathrm{eV}$ ).

\subsection{Observational study and quality control samples}

Samples were collected in the framework of an observational study involving 19 male non-hospitalized patients diagnosed with hypogonadism and other related secondary sexual disorders (one of them, 25 years old, was diagnosed with retarded puberty). Each subject gave informed consent in writing. All patients were between 25 and 79 years old and none of them has undergone other hormonal replacement therapies in the 35 days before the observational study. T was administered in the form of Tostrex Gel $2 \%{ }^{\circledR}$ (ProStrakan Ltd, Tredegar, UK) containing $20 \mathrm{mg}$ T per gram of gel. Urine samples were self-collected by each patient immediately before, 2-3, and $24 \mathrm{~h}$ after the administration using sterile Falcon tubes. Each subject took 4 puff of Tostrex Gel for an approximate single total dose of $40 \mathrm{mg} \mathrm{T}$ (bioavailability ranging from 7 to $10 \%$ ). In order to comply with the physiological circadian rhythm of patients, the drug was administered in the morning. In addition, to have a complete overview on each subject, all information regarding age, comorbidity, basal temperature and previous substitutive hormonal therapies were collected. Additional urine samples were obtained by two healthy volunteers (aged 24 and 27 years) and used for the preparation of blank and spiked quality controls. All samples were stored at $-20{ }^{\circ} \mathrm{C}$ until analysis.

\subsection{Batch analysis}

The analytical batch was composed of study samples interspersed by two different sets of quality controls as to cover at least $10 \%$ of the entire sequence (for a total of twelve injections for each set). Quality Controls 1 (QC1) consisted of samples obtained by healthy male volunteers (between 24 and 27 years) prepared according to the minimal sample preparation procedure previously described. The same samples were spiked with anabolic steroids metabolites within male physiological urinary range and analyzed as Quality Controls 2 (QC2). Before analysis, a set of ten QC1 was injected to condition and equilibrate the system. Quality control data were processed and analyzed in order to evaluate retention time drift and mass spectrometric variability. All samples were analyzed in duplicate.

\subsection{Data processing and statistical analysis, metabolite identification}

Raw data were pre-processed on large scale in a semiautomated pipeline developed in the KNIME Analytics Platform (Konstanz, Germany) [44] implemented with openMS nodes [43] (see Figure S-1 for a detailed schematization of the data processing and analysis workflow). Input data were internally recalibrated and LC-MS features detected with FeatureFinderMetabo [45]. The following conditions, deducted from the analysis of quality controls spectra in Mass Hunter Qualitative Analysis B07 (Agilent Technologies, San José, CA, USA) (see below), were used for features detection: noise intensity threshold 1500 counts, mass error $10 \mathrm{ppm}$ with dynamic re-estimation of $\mathrm{m} / \mathrm{z}$ variance during mass trace collection, isotope model set as "general metabolites" and isotope noise model with 5\% RMS error. The integration was iteratively repeated for all samples in a loop-architected workflow. Retention time was corrected considering 0.15 min of variability. Detected features were then merged in a consensus map for retention time alignment and filtered in the range 2-14.2 min and $300-850 \mathrm{~m} / \mathrm{z}$. Preliminary putative annotation against the Human Metabolome Database (www.hmdb.ca) was performed at $10 \mathrm{ppm}$ mass error considering the $[\mathrm{M}-\mathrm{H}]$ and other major expected adducts $([\mathrm{M}-\mathrm{H}],[\mathrm{M}+\mathrm{CH} 2 \mathrm{O} 2-\mathrm{H}],[\mathrm{M}+\mathrm{Na}-2 \mathrm{H}],[\mathrm{M}+\mathrm{Cl}],[\mathrm{M}-2 \mathrm{H}]$, $[\mathrm{M}-3 \mathrm{H}],[\mathrm{M}+\mathrm{K}-2 \mathrm{H}],[2 \mathrm{M}-\mathrm{H}],[2 \mathrm{M}+\mathrm{CH} 2 \mathrm{O} 2-\mathrm{H}])$. The resulting dataset was exported for further pre-treatment in Matlab. Briefly, features with more than a defined threshold for missing values (normally set at $80 \%$ ) were removed and the remaining ones replaced by a value equal to the noise threshold (1500 counts). Samples were normalized for the total signal, log transformed, mean centered and divided by the standard deviation of each variable (z-scored). The final dataset was explored with uni- and multi-variate analysis in $\mathrm{R}$ and Matlab. For multiple $t$-test we considered the q-values which give a more accurate indication of the level of false positives when testing dataset with high number of features [46]. The significance cut-off was set at 0.05 . In addition, the dataset was explored with Principal Component Analysis (PCA) and Partial Least Squares Discriminant Analysis (PLS-DA). Features with Variable Important in Projection (VIP) values $>1$ in the PLS-DA model and q-value $<0.05$ were isolated. Among these, we considered only those exhibiting increase. This subset was further 
identified on the basis of $m / z$ value and putative annotation against both HMDB and scientific literature, retention time, isotopic pattern and MS/MS fragmentation. Feature identity was confirmed by comparison with data obtained for appropriate standards, when available in our lab. MS/MS spectra were analyzed using MassHunter Qualitative Analysis B07.

\section{Results and discussion}

\subsection{Sample pre-treatment and UHPLC-MS method optimization for non-targeted analysis}

We initially aimed at obtaining a non-targeted method ensuring the detection of steroidal urinary metabolites of doping and clinical relevance in the context of the global metabolic repertoire of the sample. First injections were performed with 10 -fold sample dilution in acetonitrile/water $10 / 90 \mathrm{v} / \mathrm{v}$. Under these conditions we detected a limited number of features and, among steroidal metabolites of interest, only Andro and Etio glucuronides and sulfates were evident (present in urine at high physiological concentrations, $>2000 \mathrm{ng} / \mathrm{mL}$ [47]). In an effort to optimize sample preparation, in order to increase the range of steroids covered by the method, we characterized the chromatographic and dynamic response and signal-to-noise ratio value for selected steroidal metabolites included both in the ABP and among those of diagnostic relevance in the interpretation of the steroidal profile in clinical practice. Figure S-2 shows the extracted ion chromatograms for selected standards; Table S-1 reports their relative order of elution and mass to charge values for $[\mathrm{M}-\mathrm{H}]$ ions. Different concentrations of standards (5-2000 ng/mL, referred to the unconjugated steroid) spiked in $100 \mu \mathrm{L}$ acetonitrile/water $10 / 90 \mathrm{v} / \mathrm{v}$ were injected. For TG, we found linear response $\left(R^{2}>0.99\right)$ in the entire investigated range $(5-2000 \mathrm{ng} / \mathrm{mL})$ and signal to noise ratio higher than 3 , starting from $5 \mathrm{ng} / \mathrm{mL}$. Identical results were obtained for EG, EtioG, AndroG-d4, 5ßAdiolG-d3, AndroS, AndroS-d4, DHEAS (Figure S-3). Comparing these evidences with physiological urinary ranges reported for a population of healthy Caucasian men [47], 5-fold sample dilution, undiluted and 5-, 10-fold concentration were tested. From the analysis of obtained mass spectra and taking into account the future application of the method also for the analysis of urine collected from hypogonadal subjects, we finally opted for a minimal sample preparation with normalization of the volume of undiluted samples according to specific gravity before evaporation and reconstitution in $100 \mu \mathrm{L}$ acetonitrile/water $10 / 90 \mathrm{v} / \mathrm{v}$.

Steroids currently included in the ABP can be grouped in three isomeric couples: T/E, Andro/Etio, 5 $\alpha$ Adiol/5ßAdiol (as sum of free steroids and their $\mathrm{G}$ conjugated form, normally representing $>90 \%$ of the total excretion). In order to limit the co-elution of these analytes and other isobars, different chromatographic gradients and column temperatures were tested, starting from what reported in previous work $[40,41,48]$. In the final method, the column was set at $30^{\circ} \mathrm{C}$ and the elution was performed in gradient, starting from a mobile phase composition (acetonitrile/water $15 / 85 \mathrm{v} / \mathrm{v}$ ) similar to the sample reconstitution solution (acetonitrile/water 10/90 v/v), and gradually increasing the \% of organic phase until $95 \%$ in $14 \mathrm{~min}$, followed by rapid re-equilibration for a total run time of $16.5 \mathrm{~min}$. Representative total ion chromatogram and chromatographic separation for AndroG/EtioG and related S forms obtained under these settings are reported in Figures S-4, S-5.

Mass spectrometric conditions (nozzle, fragmentor, skimmer voltage) were optimized for ESI (-) ionization of conjugated steroids. In particular, visual analysis of mass spectra obtained for single steroidal standards revealed the formation of dimeric steroid ions $[2 \mathrm{M}-\mathrm{H}]$ (Figure S-6). In order to reduce these signals in favor of the $[\mathrm{M}-\mathrm{H}]$ molecular ion, the fragmentor voltage was varied, finding a good compromise at $200 \mathrm{~V}$. The chromatographic and mass spectrometric conditions described herein allowed to maximize the number of informative detected features in a total chromatographic run time of $16.5 \mathrm{~min}$, minimizing sample pretreatment and noise deriving from matrix interferences.

\subsection{Data pretreatment optimization and quality control analysis}

The reliability of LC-MS based metabolomics can be jeopardized by retention time drift and variations in mass accuracy during the sequence. These effects become particularly relevant when dealing with the analysis of large size batches and can cause errors in data pre-processing, features merging and annotation. In order to cope with this issue, retention time relative standard deviation (RSD) and mass to charge RSD for steroidal internal standards in quality control urine samples (QC), injected after equilibration of the system and approximately after every 10 study samples (for a total of 12 QC replicates), were evaluated (Table S-2, Figure S-7). Based on these evidences, raw data were pre-processed considering $10 \mathrm{ppm}$ mass error for peak piking, isotope merging and annotation, and 0.15 min retention time tolerance for peak alignment. Of note, the method also allowed to discriminate between the Andro G (or Etio $\mathrm{G}$ ) $[\mathrm{M}-\mathrm{H}]$ ion and the $[\mathrm{M}-\mathrm{H}+2]$ isotopologue of $5 \alpha$ Adiol $\mathrm{G}$ (or $5 \beta$ Adiol G), which elute at different retention times. After preprocessing mass spectra for QC samples collected from two volunteers (analyzed as multiple technical replicates) using these settings we detected approximately 800 individual features. These entries were putatively annotated against the Human Metabolome Database considering generally reported adducts for ESI (-) ionization mode. With these settings, $>60 \%$ of detected features received a preliminary putative annotation. Data underwent further dimensionality reduction by eliminating all features with $>80 \%$ missing values, obtaining a dataset composed of approximately 320 entries. Furthermore, to obtain an estimation of the reliability in measured abundances, we considered a set of twelve replicates for the same urine sample. Coefficient of variation \% (CV $\%$ ) (repeatability, $n=12$ ) was in the range $\pm 30 \%$ for $>75$ features, with median value at $\pm 10 \%$ (Figure S-8). Among these compounds, the steroidal $G$ and $S$ conjugates reported in Table S-3 were putatively annotated. Of note, we often obtained identical putative annotation for features at different retention times. We therefore attributed these features to steroidal isomeric forms and other isobars not currently described in the human metabolome database (as, for example, in the case of the four isomeric forms of cortolone $\mathrm{G}$ ) and trusted putative annotations only to reach a preliminary overview on the metabolic coverage of the method. Moreover, we also detected features with putative annotation corresponding to the steroidal metabolites recently proposed as additional urinary markers of testosterone intake (e.g., 6 $\beta-\mathrm{OH}$-androsterone-3-

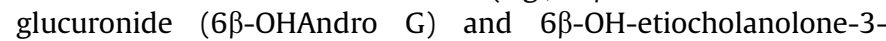
glucuronide (6ß-OHEtio G) [49]).

As proof of concept, we applied the pipeline for the comparative analysis of a blank quality control group (QC1) and a second group obtained spiking the same urine with endogenous steroids within urinary physiological concentration ranges previously reported for the male Caucasian population (QC2) (Table 1) [47]. The data matrix obtained by processing with OpenMS was pretreated as above described (missing values replaced by the noise threshold (1500 counts), normalized for total signal, log transformed and z-scored) and explored with principal component analysis (PCA). PCA allowed a preliminary exploration of the dataset highlighting outliers (excluded from further analysis) and straightforward QC1 and QC2 spontaneous groups clustering (Fig. 3A). In order to stress out the separation between observed groups and rank features most responsible for clustering, data were further investigated in a 
Table 1

VIP scores obtained for steroids spiked in quality control samples, referred to the first component of the PLS-DA model.

\begin{tabular}{lll}
\hline Compound & $\begin{array}{l}\text { Spiked concentration (ng/ } \\
\mathrm{mL})\end{array}$ & $\begin{array}{l}\text { VIP } \\
\text { score }\end{array}$ \\
\hline $\begin{array}{l}\text { Dehydroisoepiandrosterone } \\
\quad \text { glucuronide }\end{array}$ & 2000 & 2.6 \\
$\begin{array}{l}\text { Androsterone sulfate } \\
\text { Etiocholanolone glucuronide }\end{array}$ & 2000 & $>3$ \\
Testosterone glucuronide & 2000 & $>5$ \\
Epitestosterone glucuronide & 100 & $>3$ \\
\hline
\end{tabular}

Partial Least Squares - Discriminant Analysis model (PLS-DA). In contrast to PCA, PLS-DA maximizes the variance existent in the dataset using the a priori knowledge of class assignments. Obtained scores plot for PLS-DA first three components computed for QC1 and QC2 data is reported in Fig. 3B. Component one explained approximately $18 \%$ of the entire variability, whereas component two and three were less explicative. Therefore, we considered the ranking list for variables important in projection (VIP) related to component 1 . These values account for the amount of explained variation in each dimension in regard to single entries of the dataset. As showed in Table 1, VIP values for all steroids spiked in the QC2 group exhibit scores $>1$. Concerning other features significantly increased in the QC2 group and with VIP $>1$, we found some adducts of the spiked steroids corresponding to the sodium formate cluster $([\mathrm{M}+\mathrm{HCOONa}-\mathrm{H}])$. These adducts were confirmed analyzing MS spectra obtained for pure standards (Figure S-11).

Eventually, when applied for the analysis of quality control samples obtained by single spot urine collection from two volunteers, the here described method allows the detection of approximately 800 features and a preliminary putative annotation of $>60 \%$ of them against the Human Metabolome Database, considering $10 \mathrm{ppm}$ error and main ESI (-) reported adducts. Among these features, steroids of clinical and doping relevance are detectable with high reliability on measured ion abundance, making the method appropriate also for relative metabolite quantification. In addition, PLS-DA analysis and VIP ranking revealed the influence in group clustering of steroids spiked in the QC2 group and the suitability of this approach for the comparative investigation of the urinary steroidal profile of Caucasian men in the context of the urinary metabolic repertoire.

\subsection{Comparative analysis of urines after single transdermal testosterone administration}

To explore the applicability of the non-targeted workflow, we investigated the metabolic signature of urines collected in the framework of an observational study involving 19 males in treatment with transdermal $\mathrm{T}$. Topical formulations of $\mathrm{T}$ are generally indicated in the therapy of common pathological conditions including male hypogonadism and other secondary sexual disorders. In general, these pharmaceutical preparations are considered a gold standard in all those cases in which local action and limited systemic adsorption are advisable. Due to the low relative bioavailability, modest modifications of the urinary steroidal profile are normally expected after treatment. This study involved nonhospitalized patients between 25 and 79 years old (median age 62 ), diagnosed with Latent Onset Hypogonadism syndrome ( $\mathrm{LOH}$ ) and related secondary sexual disorders. Testosterone was self-
A

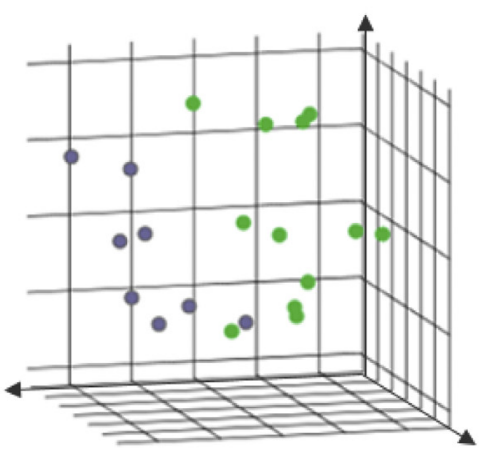

C

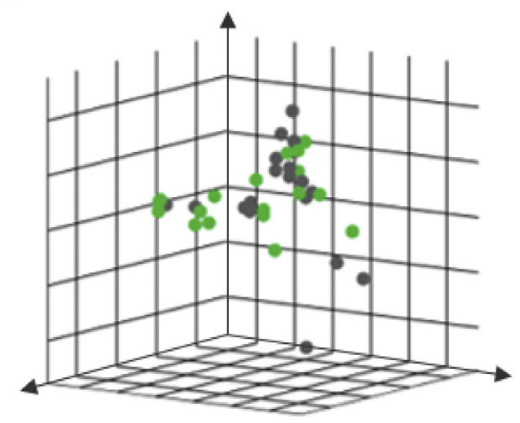

B

\section{PLS-DA}

GROUP
QC1
QC2

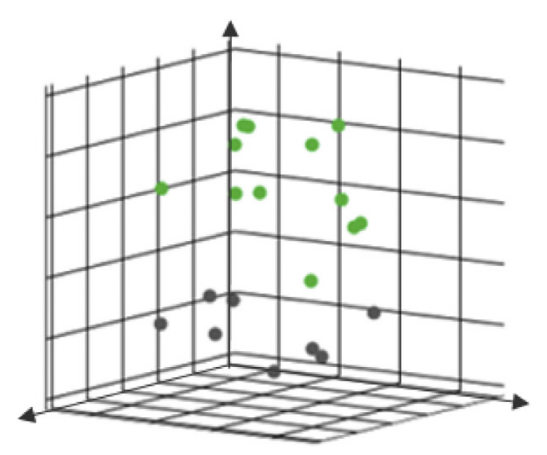

D
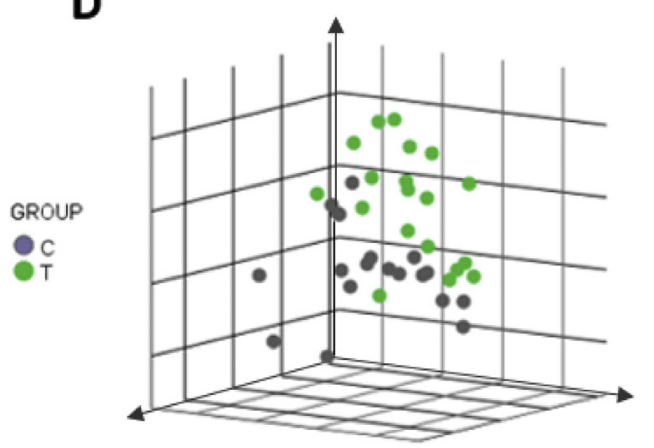

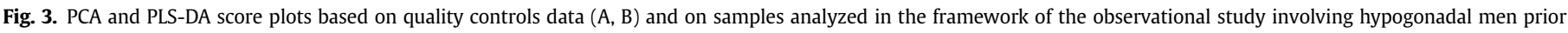
(control group) and after (treatment group) single testosterone gel administration (C, D). 
administered topically, between 09:00 and 10:00 in the morning, for a single total dose of $40 \mathrm{mg}$ (bioavailability from 7 to $10 \%$ ). Urine collection started immediately before the application (control), followed by sampling at approximately $2-3 \mathrm{~h}$ (treatment) and $24 \mathrm{~h}$ (elimination) after the intake. Samples were divided in three groups according to the expected excretion profile. MS analysis and processing of the urine samples lead to the identification of $>3000$ metabolic features, of which $>60 \%$ putatively annotated.

Considering the intrinsic high inter-individual variability of the population under investigation due to relevant difference in age and the presence of diverse comorbidities (including, but not limited to, diabetes, hypertension, leukemia, depression), and in view of the possible detection of metabolites only after treatment, the dataset was reduced by eliminating features with $>90 \%$ missing values. The resulting 1100 features were normalized and scaled. The coefficient of variation \% for technical replicates was found included in the range $\pm 30 \%$ for approximately 300 features in each sample, with median value $<10 \%$ (Figure S-9). Preliminary exploration of the obtained multidimensional dataset with PCA failed in underline any clustering and confirmed the expected broad metabolic inter-individual variability (Fig. 3C). In such confused data, supervised multivariate methods can provide valuable help in isolating only the informative fraction from high-dimensional datasets. In this regard, PLS-DA modeling and VIP scores analysis returned the expected group clustering (Fig. 3D). All features exhibiting VIP $>1$ (referred to the first score component, explaining approximately $10 \%$ of the total variance) were isolated. This subset was further filtered i) retaining only features increased after treatment and ii) responding to a significance threshold set at 0.05 for $\mathrm{p}$-values corrected for multiple testing (q-values). Each feature in agreement with these criteria was confirmed by visual analysis of mass spectra and evaluation of the short-term excretion profile considering control, treatment and elimination groups. This procedure led to the list of metabolites reported in Table 2.

Putative annotation of these compounds was confirmed in MS/ MS experiments underlining the loss of the glucuronic group and the formation of fragments previously reported for steroidal conjugates [50,51] or present in in silico predicted spectra [52] (see Table S-4). For TG and EG, both fragmentation and retention times were also consistent with data obtained analyzing pure standards (Figure S-11). The possibility of the detection of two isotopic forms deriving from the same metabolite for features co-eluting at $7.5 \mathrm{~min}(\mathrm{~m} / \mathrm{z}$ of 541.265 and 539.250$)$ was excluded by the analysis of the isotopic pattern (Figure S-10). Beyond putative annotations

Table 2

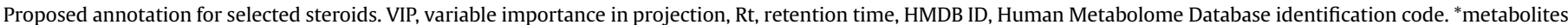
only putatively annotated.

\begin{tabular}{|c|c|c|c|c|c|c|c|}
\hline VIP score & Measured $[\mathrm{M}-\mathrm{H}] \mathrm{m} / \mathrm{z}$ & Calculated $[\mathrm{M}-\mathrm{H}] \mathrm{m} / \mathrm{z}$ & Error (ppm) & Rt (min) & & Annotation & HMDB ID \\
\hline \multirow[t]{2}{*}{2.83} & 367.1009 & 367.1035 & 6.8 & 3.8 & M1 & Caffeoyl methylquinic acid* & HMDB39960 \\
\hline & & & & & & Ferulylquinic acid* & HMDB30669 \\
\hline \multirow[t]{2}{*}{1.79} & 367.1005 & 367.1035 & 8.1 & 4.0 & M2 & Caffeoyl methylquinic acid* & HMDB39960 \\
\hline & & & & & & Ferulylquinic acid* & HMDB30669 \\
\hline 1.82 & 439.1591 & & & 6.8 & M3 & - & - \\
\hline \multirow[t]{6}{*}{1.87} & 541.2613 & 541.2654 & 7.6 & 6.8 & & $20 \alpha$-Cortolone glucuronide & HMDB10320 \\
\hline & & & & & & $20 \beta$-Cortolone glucuronide & \\
\hline & & & & & & $20 \alpha, 5 \alpha$-Cortolone glucuronide & \\
\hline & & & & & & $20 \beta, 5 \alpha$-Cortolone glucuronide & \\
\hline & & & & & & Tetrahydrocortisol glucuronide & \\
\hline & & & & & & $5 \alpha$-Tetrahydrocortisol glucuronide & \\
\hline \multirow[t]{6}{*}{1.49} & 541.2656 & 541.2654 & 0.4 & 7.0 & & $20 \alpha$-Cortolone glucuronide & HMDB10320 \\
\hline & & & & & & $20 \beta$-Cortolone glucuronide & \\
\hline & & & & & & $20 \alpha, 5 \alpha$-Cortolone glucuronide & \\
\hline & & & & & & $20 \beta, 5 \alpha$-Cortolone glucuronide & \\
\hline & & & & & & Tetrahydrocortisol glucuronide & \\
\hline & & & & & & $5 \alpha$-Tetrahydrocortisol glucuronide & \\
\hline 2.17 & 445.2001 & 445.1994 & 1.6 & 7.2 & M4 & Olmesartan* & HMDB14420 \\
\hline \multirow[t]{6}{*}{1.52} & 541.2623 & 541.2654 & 5.7 & 7.5 & & $20 \alpha$-Cortolone glucuronide & HMDB10320 \\
\hline & & & & & & 20 $\beta$-Cortolone glucuronide & \\
\hline & & & & & & $20 \alpha, 5 \alpha$-Cortolone glucuronide & \\
\hline & & & & & & $20 \beta, 5 \alpha$-Cortolone glucuronide & \\
\hline & & & & & & Tetrahydrocortisol glucuronide & \\
\hline & & & & & & $5 \alpha$-Tetrahydrocortisol glucuronide & \\
\hline \multirow[t]{6}{*}{1.82} & 541.2650 & 541.2654 & 0.7 & 7.9 & & $20 \alpha$-Cortolone glucuronide & HMDB10320 \\
\hline & & & & & & $20 \beta$-Cortolone glucuronide & \\
\hline & & & & & & $20 \alpha, 5 \alpha$-Cortolone glucuronide & \\
\hline & & & & & & $20 \beta, 5 \alpha$-Cortolone glucuronide & \\
\hline & & & & & & Tetrahydrocortisol glucuronide & \\
\hline & & & & & & $5 \alpha$-Tetrahydrocortisol glucuronide & \\
\hline \multirow[t]{2}{*}{1.39} & 539.2510 & 539.2497 & 2.4 & 7.5 & & Tetrahydrocortisone glucuronide & - \\
\hline & & & & & & Tetrahydroaldosterone glucuronide & HMDB10357 \\
\hline 1.68 & 509.2662 & & & 7.5 & M5 & - & \\
\hline \multirow[t]{2}{*}{1.65} & 479.2192 & 479.2286 & 7.3 & 7.9 & & 11-Oxo-androsterone glucuronide & HMDB10338 \\
\hline & & & & & & 11-Oxo-etiocholanolone glucuronide & \\
\hline \multirow[t]{4}{*}{1.29} & 481.2436 & 481.2443 & 1.5 & 8.2 & & $11 \beta$-Hydroxyandrosterone glucuronide & HMDB10351 \\
\hline & & & & & & $11 \beta$-Hydroxyetiocholanolone glucuronide & \\
\hline & & & & & & $6 \beta$-Hydroxyandrosterone glucuronide & \\
\hline & & & & & & $6 \beta$-Hydroxyetiocholanolone glucuronide & \\
\hline 2.4 & 511.2501 & & & 8.3 & M6 & - & - \\
\hline \multirow[t]{2}{*}{1.83} & 479.2251 & 479.2287 & 7.5 & 8.8 & & 11-Oxo-androsterone glucuronide & HMDB10338 \\
\hline & & & & & & 11-Oxo-etiocholanolone glucuronide & \\
\hline 1.58 & 463.2280 & 463.2337 & 5.6 & 9.5 & & Testosterone glucuronide & HMDB03193 \\
\hline 1.97 & 463.2293 & 463.2337 & 9.5 & 10.5 & & Epitestosterone glucuronide & - \\
\hline
\end{tabular}




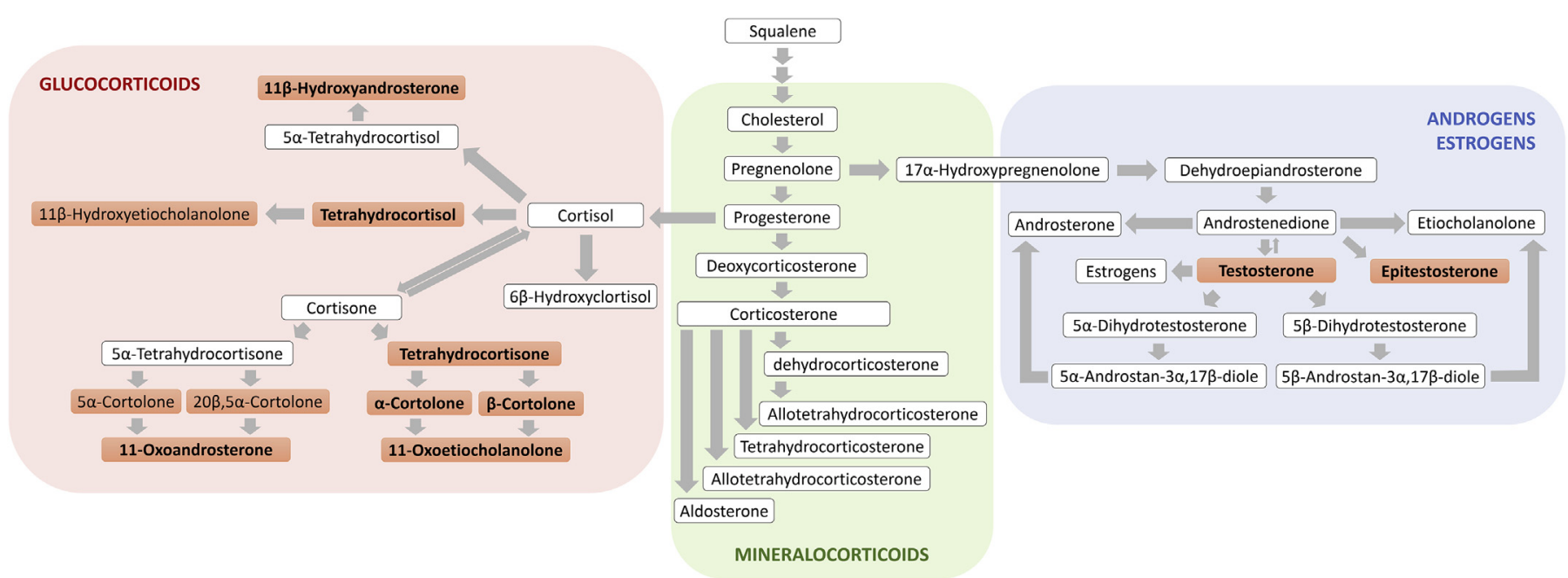

EXCRETED IN URINE AS GLUCURONO- AND SULFO-CONJUGATES

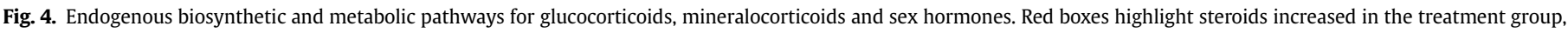

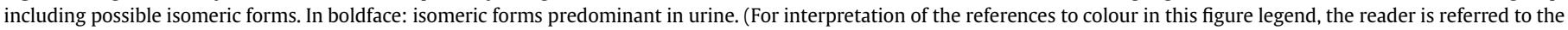
web version of this article.)

obtained from the Human Metabolome Database, we noticed several isomeric forms of the isolated features not reported in this repository. Notwithstanding, this point represented a limiting aspect for data analysis and required further time-consuming literature investigation. Anyway, we tried to obtain valuable information in order to discriminate between detected peaks, considering previously reported isomers relative abundance in urine $[53,54]$. On this basis we derived the final annotations reported in Table 2 (and Table S-4) and Fig. 4.

The most remarkable metabolic signature characterizing the treatment group resulted in an increase in the urinary excretion of steroids in the anabolic androgenic cascade (TG and EG), together with the final metabolic products of the glucocorticoid biosynthetic pathway (Fig. 4). All these metabolites showed similar short-term excretion profiles (Fig. 5), increasing in the treatment group and decreasing in the predicted elimination phase. However, it has to be underlined that in the context of the observational study involving non-hospitalized men, it was not possible to withhold the subjects for more than one day. Consequently, the groups of samples investigated also show significant circadian steroidal fluctuations. In particular, it is well established that cortisol (F) and cortisone biosynthesis are subject to significant circadian regulation. A related trend has been reported also for some of their metabolic products in urine (tetrahydrocortisol G (THFG), 5 $\alpha$-tetrahydrocortisol G ( $5 \alpha$-THFG), tetrahydrocortisone G (THEG), $5 \alpha$-tetrahydrocortisone $\mathrm{G}$ ( $5 \alpha$-THEG), and derived four isomers of cortolone $G)$ [55]. In particular, in healthy men blood $F$ concentration reaches a maximum around 9:00 a.m., while the related urinary metabolites exhibit the peak about $2 \mathrm{~h}$ later. Of note, the treatment group was collected approximately around noon, this meaning in concomitance with the acrophase for the excretion of $\mathrm{F}$ metabolites in urine. The short-term excretion profile (Fig. 5 A,C) supports this interpretation. Interestingly, we also noticed a significant increase in the excretion of 11-oxo-etiocholanolone G (11-oxoEtioG) and 11oxo-androsterone $\mathrm{G}$ (11-oxoAndroG), both final urinary metabolites derived from $\mathrm{F}$ and cortisol (Figs. 4 and $5 \mathrm{~A}, \mathrm{C}$ ) even if for these metabolites it has been previously reported no significant circadian behavior in urine collected from healthy volunteers [55]. We also detected an increase in metabolic features annotated in the HMDB as $11 \beta$-hydroxyandrosterone G (11ß-OHAndroG) and $11 \beta$ - hydroxyetiocholanolone $\mathrm{G}$ (11ß-OHEtioG). Of note, these metabolites are also consistent with the non-hydrolyzable glucuronated forms of $6 \beta$-OHAndro and $6 \beta$-OHEtio, recently reported as valuable urinary markers providing extended detection window for oral $\mathrm{T}$ administrations [49]. This observation also gives additional evidence on the importance to perform additional studies to clarify the utility of these novel markers also in the detection of topical T intake, and supports their further evaluation for the inclusion in the steroidal module of the ABP.

Although our findings are likely to be direct consequence of the transdermal administration of $\mathrm{T}$ gel and are consistent with the evidence reported in previous studies [30], we believe that for an unambiguous interpretation of these data further studies have to be specifically designed. In particular, larger cohorts are needed to correct for the physiological circadian variability.

Beyond these modifications of the urinary steroidal profile, we isolated three significantly increased metabolic features (M3, M4, M5, see Table 2), with short term excretion profile consistent with what expected after single administration of the steroidal formulation (Fig. 5 D). Although no putative annotation was obtained, we detected the related MS/MS fragments indicating the presence of the glucuronic group (Table S-4), this supporting the possibility to be associated to glucurono-conjugated metabolites of steroidal compounds. Table S-4 reports the hypothesized molecular formulas, computed considering the measured $\mathrm{m} / \mathrm{z}$ values, isotopic patterns and fragment ions relative abundances.

We also observed the putative increase of dietary components (M1 and M2, caffeoyl methylquinic acid or ferulylquinic acid) and of olmesartan (M4), an angiotensin II receptor antagonist indicated in the treatment of hypertension (Table 2). Taking into account the median age of the population of patients involved in the study (about 62 years) and the presence of hypertension as comorbidity state in the majority of these subjects, we infer the use of this drug in the context of chronic therapeutic administrations, probably in the morning, this explaining its presence in the group of samples collected around 12:00 a.m.

Based on these evidences, the non-targeted workflow herein developed provided us with a satisfactory portrait of the metabolic signature of samples collected in the context of an observational study characterized by high inter-individual metabolic variability. 


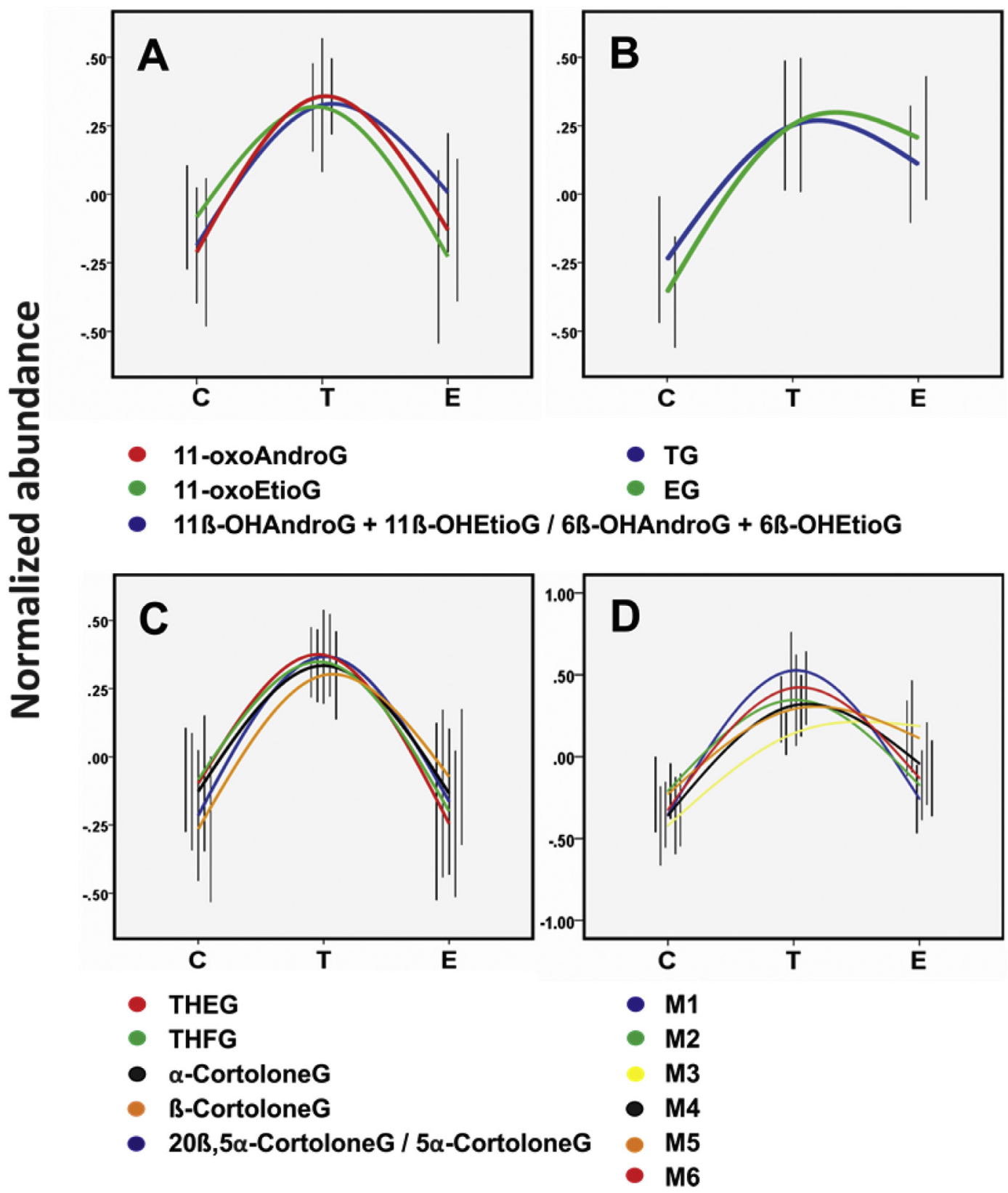

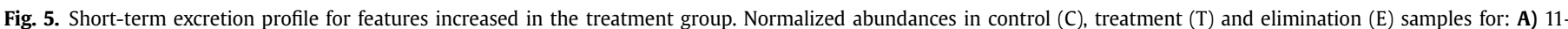

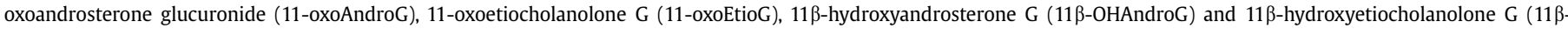

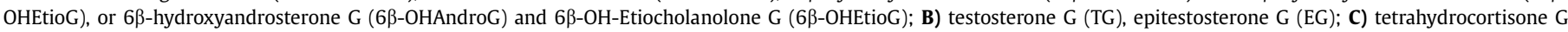

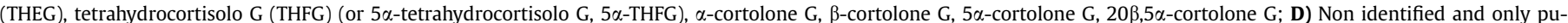
tatively annotated metabolites (see Table 2 and Table S-3 for description). Error bars indicate \pm 1 standard error.

Despite the complexity of the population under investigation and the expected slight modifications of the steroidal profile as consequence of single transdermal steroidal application, our approach still succeeded in raising insights about most involved metabolic pathways and in isolating previously not reported metabolic features that correlate with samples clusters.

\section{Conclusions}

In this work we described a liquid-chromatography mass spectrometry based metabolomics workflow optimized for the non-targeted analysis of the urinary steroidal profile in the context of the whole metabolic content of the sample. This method allows the detection of up to $>3000$ individual metabolic features among witch $>60 \%$ preliminary putatively annotated. For approximately 300 of them in each sample high reliability on measured ion abundances was gauged, making the method germane also for relative comparison of metabolites urinary levels. In addition, it ensures sensitivity for steroids of doping and clinical relevance, directly detected in the form of $\mathrm{G}$ and $\mathrm{S}$ conjugates, within physiological urinary concentration ranges and after a naïve pretreatment of approximately $100 \mu \mathrm{L}$ of urine.

When applied for the analysis of a real cohort of urine samples, the analytical and data analysis pipeline successfully delineates the metabolic signature of each group, mainly due to circadian steroidal fluctuations and to the increase in androgen metabolites 
excretion. Noteworthy, it also permits the straightforward detection of other unexpected and previously unknown steroidal metabolic features, contributing to the available reference background for future studies focused on the implementation of the currently adopted urinary steroidal markers.

More in general, both forensic and clinical research can take advantage of such holistic methodology as first line exploratory strategy for the analysis of samples obtained in in vitro metabolism studies and in vivo controlled administration trials. Indeed, compared to classical targeted approaches (Fig. 1), non-targeted analysis substantially extends the perspective moving the attention from isolated metabolic modifications towards a more defined metabolic picture.

However, despite the great potentiality in biomarker discovery, when dealing with the accurate validation of selected candidates neither targeted nor non-targeted metabolomics can provide results not constrained by the availability of an adequate population of samples in terms of i) number, ii) time of collection (e.g. in order to circumvent circadian fluctuations) and iii) inclusion criteria for individuals in the study cohort. These aspects prominently affect the amount of undesired variability and reduce the isolable informative fraction from acquired data.

Furthermore, when coming to the non-targeted analysis of steroidal metabolites including preliminary annotation against currently available metabolite repositories, it has to be taken into account that even if the number of metabolites included in these databases is impressive, it does not yet cover the entire available knowledge about human metabolism. In particular, in the case of the urinary steroidal profile we found several isomeric forms and $G$ and $\mathrm{S}$ conjugates thereof, not reported in databases but broadly described in literature. This represents a time consuming bottleneck in metabolomics and suggests that a critical interpretation of obtained data on the basis of currently available knowledge remains an important step whenever high level of accuracy and solidity is required.

\section{Acknowledgments}

We are grateful to Prof. Francesco Romanelli and Dr. Massimiliano Sansone, Department of Experimental Medicine, Sapienza University of Rome, for providing the anonymized samples from the observational study.

\section{Appendix A. Supplementary data}

Supplementary data related to this article can be found at http:// dx.doi.org/10.1016/j.aca.2017.01.055.

\section{Summary of abbreviations}

$\begin{array}{ll}\text { T } & \text { Testosterone } \\ \text { E } & \text { Epitestosterone } \\ \text { Andro } & \text { Androsterone } \\ \text { Etio } & \text { Etiocholanolone } \\ 5 \alpha \text { Adiol } & 5 \alpha \text {-androstane-3 } \alpha, 17 \beta \text {-diol } \\ 5 \beta \text { Adiol } & 5 \beta \text {-androstane- } \alpha, 17 \beta \text {-diol } \\ \text { DHEA } & \text { Dehydroisoandrosterone } \\ \text { F } & \text { Cortisol } \\ \text { THF } & \text { Tetrahydrocortisol } \\ \text { THE } & \text { Tetrahydrocortisone } \\ \alpha \text { THF } & 5 \alpha \text {-tetrahydrocortisol } \\ \alpha \text { THE } & 5 \alpha \text {-tetrahydrocortisone }\end{array}$

11-oxoEtio 11-oxo-etiocholanolone

11-oxoAndro 11-oxo-androsterone

$11 \beta$-OHEtio 11 $\beta$-hydroxyetiocholanolone
$11 \beta$-OHAndro $11 \beta$-hydroxyandrosterone

$6 \beta$-OHEtio 6 $\beta$-hydroxyetiocholanolone

$6 \beta$-OHAndro $6 \beta$-hydroxyandrosterone

G Glucuronide

S Sulfate

\section{References}

[1] M.P. Caulfield, T. Lynn, M.E. Gottschalk, K.L. Jones, N.F. Taylor E.M. Malunowicz, C.H. Shackleton, R.E. Reitz, D.A. Fisher, The diagnosis of congenital adrenal hyperplasia in the newborn by gas chromatography/mass spectrometry analysis of random urine specimens, J. Clin. Endocrinol. Metab. 87 (2002) 3682-3690, http://dx.doi.org/10.1210/jcem.87.8.8712.

[2] N.F. Taylor, Urinary steroid profiling, Methods Mol. Biol. 1065 (2013) 259-276, http://dx.doi.org/10.1007/978-1-62703-616-0_17.

[3] N. Krone, B.A. Hughes, G.G. Lavery, P.M. Stewart, W. Arlt, C.H.L. Shackleton, Gas chromatography/mass spectrometry (GC/MS) remains a pre-eminent discovery tool in clinical steroid investigations even in the era of fast liquid chromatography tandem mass spectrometry (LC/MS/MS), J. Steroid Biochem. Mol. Biol. $121 \quad$ (2010) 496-504, http://dx.doi.org/10.1016/ j.jsbmb.2010.04.010.

[4] C.H.L. Shackleton, Mass spectrometry in the diagnosis of steroid-related disorders and in hypertension research, J. Steroid Biochem. Mol. Biol. 45 (1993) 127-140, http://dx.doi.org/10.1016/0960-0760(93)90132-G.

[5] C.H.L. Shackleton, Profiling steroid hormones and urinary steroids, J. Chromatogr. B Biomed. Sci. Appl. 379 (1986) 91-156, http://dx.doi.org/ 10.1016/S0378-4347(00)80683-0.

[6] Y. Koyama, K. Homma, T. Hasegawa, Urinary steroid profiling: a powerful method for the diagnosis of abnormal steroidogenesis, Expert Rev. Endocrinol. Metab. 9 (2014) 273-282, http://dx.doi.org/10.1586/17446651.2014.904199.

[7] G.G. Lavery, J. Idkowiak, M. Sherlock, I. Bujalska, J.P. Ride, K. Saqib, M.F. Hartmann, B. Hughes, S.A. Wudy, J. De Schepper, W. Arlt, N. Krone, C.H. Shackleton, E.A. Walker, P.M. Stewart, Novel H6PDH mutations in two girls with premature adrenarche: "apparent" and "true" CRD can be differentiated by urinary steroid profiling, Eur. J. Endocrinol. 168 (2013), http:// dx.doi.org/10.1530/EJE-12-0628.

[8] N. Reisch, Substitution therapy in adult patients with congenital adrenal hyperplasia, Best. Pract. Res. Clin. Endocrinol. Metab. 29 (2015) 33-45, http:// dx.doi.org/10.1016/j.beem.2014.11.002.

[9] T.M.A. Kerkhofs, M.N. Kerstens, I.P. Kema, T.P. Willems, H.R. Haak, Diagnostic value of urinary steroid profiling in the evaluation of adrenal tumors, Horm Cancer 6 (2015) 168-175, http://dx.doi.org/10.1007/s12672-015-0224-3.

[10] L. Konieczna, T. Baczek, M. Belka, A. Fel, M. Markuszewski, W. Struck, M. Markuszewski, R. Kaliszan, Steroid profiles as potential biomarkers in patients with urogenital tract cancer for diagnostic investigations analyzed by liquid chromatography coupled to mass spectrometry, J. Pharm. Biomed. Anal. 73 (2013) 108-115, http://dx.doi.org/10.1016/j.jpba.2012.03.016.

[11] C.D. Rahnema, L.I. Lipshultz, L.E. Crosnoe, J.R. Kovac, E.D. Kim, Anabolic steroid-induced hypogonadism: diagnosis and treatment, Fertil. Steril. 101 (2014) 1271-1279, http://dx.doi.org/10.1016/j.fertnstert.2014.02.002.

[12] A.O.K. Chan, C.C. Shek, Urinary steroid profiling in the diagnosis of congenital adrenal hyperplasia and disorders of sex development: experience of a urinary steroid referral centre in Hong Kong, Clin. Biochem. 46 (2013) 327-334, http://dx.doi.org/10.1016/j.clinbiochem.2012.12.006.

[13] N. Meklat, J.C. Tabet, J. de Ceaurriz, Urine ratio of tetrahydrocortisol to tetrahydrodeoxycortisol to screen for the systemic administration of cortisone and hydrocortisone, Forensic Sci. Int. 185 (2009), http://dx.doi.org/10.1016/ j.forsciint.2008.12.011.

[14] The World Anti-Doping Agency, Endogenous Anabolic Androgenic Steroids Measurements and Reporting, WADA Technical Document TD2016EAAS, 2016. http://www.wada-ama.org (Accessed 06 June 2016).

[15] The World Anti-Doping Agency, The World Anti-doping Agency Code, the 2016 Prohibited List International Standard, 2016. http://www.wada-ama.org (Accessed 06 June 2016).

[16] M. Donike, K. Bärwald, K. Klostermann, W. Schänzer, J. Zimmermann, Nachwies von exogenem testosterone, in: H. Heck, W. Hollmann, H. Liesen, R. Rost (Eds.), Sport. Leistung Und Gesundh, Deutscher Ärzte Verlag, Köln, 1983, pp. 293-298.

[17] A. Ouellet, N. Leberre, C. Ayotte, A simplified and accurate method for the analysis of urinary metabolites of testosterone-related steroids using gas chromatography/combustion/isotope ratio mass spectrometry, Rapid Commun. Mass Spectrom. 27 (2013) 1739-1750, http://dx.doi.org/10.1002/ rcm.6620.

[18] X. de la Torre, C. Colamonici, D. Curcio, F. Molaioni, F. Botrè, A comprehensive procedure based on gas chromatography-isotope ratio mass spectrometry following high performance liquid chromatography purification for the analysis of underivatized testosterone and its analogues in human urine, Anal. Chim. Acta 756 (2012) 23-29, http://dx.doi.org/10.1016/j.aca.2012.10.013.

[19] A.T. Cawley, U. Flenker, The application of carbon isotope ratio mass spectrometry to doping control, J. Mass Spectrom. 43 (2008) 854-864, http:// dx.doi.org/10.1002/jms.

[20] P.E. Sottas, M. Saugy, C. Saudan, Endogenous steroid profiling in the athlete 
biological passport, Endocrinol. Metab. Clin. North Am. 39 (2010) 59-73, http://dx.doi.org/10.1016/j.ecl.2009.11.003.

[21] M. Saugy, C. Lundby, N. Robinson, Monitoring of biological markers indicative of doping: the athlete biological passport, Br. J. Sports Med. 48 (2014) 827-832, http://dx.doi.org/10.1136/bjsports-2014-093512.

[22] P.E. Sottas, N. Robinson, O. Rabin, M. Saugy, The athlete biological passport Clin. Chem. 57 (2011) 969-976, http://dx.doi.org/10.1373/ clinchem.2011.162271.

[23] P.E. Sottas, N. Baume, C. Saudan, C. Schweizer, M. Kamber, M. Saugy, Bayesian detection of abnormal values in longitudinal biomarkers with an application to T/E ratio, Biostatistics 8 (2007) 285-296, http://dx.doi.org/10.1093/ biostatistics/kxl009.

[24] P.E. Sottas, C. Saudan, C. Schweizer, N. Baume, P. Mangin, M. Saugy, From population- to subject-based limits of T/E ratio to detect testosterone abuse in elite sports, Forensic Sci. Int. 174 (2008) 166-172, http://dx.doi.org/10.1016/ j.forsciint.2007.04.001.

[25] J.J. Schulze, J. Lundmark, M. Garle, L. Ekström, P.E. Sottas, A. Rane, Substantial advantage of a combined Bayesian and genotyping approach in testosterone doping tests, Steroids 74 (2009) 365-368, http://dx.doi.org/10.1016/ j.steroids.2008.11.003.

[26] A. Rane, L. Ekström, Androgens and doping tests: genetic variation and pitfalls, Br. J. Clin. Pharmacol. 74 (2012) 3-15, http://dx.doi.org/10.1111/j.13652125.2012.04294.x.

[27] E. Strahm, J.E. Mullen, N. Gårevik, M. Ericsson, J.J. Schulze, A. Rane, L. Ekström, Dose-dependent testosterone sensitivity of the steroidal passport and GC-CIRMS analysis in relation to the UGT2B17 deletion polymorphism, Drug Test. Anal. 7 (2015) 1063-1070, http://dx.doi.org/10.1002/dta.1841.

[28] F. Ponzetto, F. Mehl, J. Boccard, N. Baume, S. Rudaz, M. Saugy, R. Nicoli, Longitudinal monitoring of endogenous steroids in human serum by UHPLC-MS/ MS as a tool to detect testosterone abuse in sports, Anal. Bioanal. Chem. 408 (2016) 705-719, http://dx.doi.org/10.1007/s00216-015-9185-1.

[29] F. Badoud, J. Boccard, C. Schweizer, F. Pralong, M. Saugy, N. Baume, Profiling of steroid metabolites after transdermal and oral administration of testosterone by ultra-high pressure liquid chromatography coupled to quadrupole time-offlight mass spectrometry, J. Steroid Biochem. Mol. Biol. 138 (2013) 222-235, http://dx.doi.org/10.1016/j.jsbmb.2013.05.018.

[30] A. Fabregat, O.J. Pozo, P. Van Renterghem, P. Van Eenoo, J. Marcos, J. Segura, R. Ventura, Detection of dihydrotestosterone gel, oral dehydroepiandrosterone, and testosterone gel misuse through the quantification of testosterone metabolites released after alkaline treatment, Drug Test. Anal. 3 (2011) 828-835, http://dx.doi.org/10.1002/dta.351.

[31] G. Miller, V. Nair, S. Morrison, M. Summers, S. Willick, D. Eichner, Intranasal delivery of natesto testosterone gel and its effects on doping markers, Drug Test. Anal. (2016), http://dx.doi.org/10.1002/dta.2106.

[32] M. Schonfelder, H. Hofmann, T. Schulz, T. Engl, D. Kemper, B. Mayr, C. Rautenberg, R. Oberhoffer, D. Thieme, Potential detection of low-dose transdermal testosterone administration in blood, urine and saliva, Drug Test. Anal. (2016), http://dx.doi.org/10.1002/dta.2110.

[33] A. Palermo, F. Botrè, X. de la Torre, I. Fiacco, M. Iannone, M. Mazzarino, Drugdrug interactions and masking effects in sport doping: influence of miconazole administration on the urinary concentrations of endogenous anabolic steroids, Forensic Toxicol. 34 (2016) 386-397, http://dx.doi.org/10.1007/ s11419-016-0325-x.

[34] D. Thieme, P. Anielski, J. Grosse, H. Sachs, R.K. Mueller, Identification of anabolic steroids in serum, urine, sweat and hair: comparison of metabolic patterns, Anal. Chim. Acta 483 (2003) 299-306, http://dx.doi.org/10.1016/ S0003-2670(02)01604-5.

[35] X. Matabosch, O.J. Pozo, C. Pérez-Mañá, M. Farré, J. Marcos, J. Segura, R. Ventura, Discrimination of prohibited oral use from authorized inhaled treatment of budesonide in sports, Ther. Drug Monit. 35 (2013) 118-128, http://dx.doi.org/10.1097/FTD.0b013e3182787b20.

[36] X. Matabosch, O.J. Pozo, N. Monfort, C. Perez-Mañá, M. Farré, J. Marcos, J. Segura, R. Ventura, Urinary profile of methylprednisolone and its metabolites after oral and topical administrations, J. Steroid Biochem. Mol. Biol. 138 (2013) 214-221, http://dx.doi.org/10.1016/j.jsbmb.2013.05.019.

[37] Y. Wang, R. Caldwell, D.A. Cowan, C. Legido-Quigley, LC-MS-based metabolomics discovers purine endogenous associations with low-dose salbutamol in urine collected for antidoping tests, Anal. Chem. 88 (2016) 2243-2249, http://dx.doi.org/10.1021/acs.analchem.5b03927.

[38] A. Kiss, M. Lucio, A. Fildier, C. Buisson, P. Schmitt-Kopplin, C. Cren-Olivé, Doping Control Using High, Ultra-High Resolution, Mass spectrometry based non-targeted metabolomics-a case study of salbutamol and budesonide abuse, PLoS One 8 (2013), http://dx.doi.org/10.1371/journal.pone.0074584.
[39] J. Boccard, F. Badoud, E. Grata, S. Ouertani, M. Hanafi, G. Mazerolles, P. Lantéri, J.L. Veuthey, M. Saugy, S. Rudaz, A steroidomic approach for biomarkers discovery in doping control, Forensic Sci. Int. 213 (2011) 85-94, http:// dx.doi.org/10.1016/j.forsciint.2011.07.023.

[40] J. Boccard, F. Badoud, N. Jan, R. Nicoli, C. Schweizer, F. Pralong, J.-L. Veuthey, N. Baume, S. Rudaz, M. Saugy, Untargeted profiling of urinary steroid metabolites after testosterone ingestion: opening new perspectives for antidoping testing, Bioanalysis 6 (2014) 2523-2536, http://dx.doi.org/10.4155/ bio.14.200.

[41] M. Raro, M. Ibáñez, R. Gil, A. Fabregat, E. Tudela, K. Deventer, R. Ventura J. Segura, J. Marcos, A. Kotronoulas, J. Joglar, M. Farré, S. Yang, Y. Xing, P. Van Eenoo, E. Pitarch, F. Hernández, J.V. Sancho, O.J. Pozo, Untargeted metabolomics in doping control: detection of new markers of testosterone misuse by ultrahigh performance liquid chromatography coupled to high-resolution mass spectrometry, Anal. Chem. 87 (2015) 8373-8380, http://dx.doi.org/ 10.1021/acs.analchem.5b02254.

[42] F. Jeanneret, D. Tonoli, M.F. Rossier, M. Saugy, J. Boccard, S. Rudaz, Evaluation of steroidomics by liquid chromatography hyphenated to mass spectrometry as a powerful analytical strategy for measuring human steroid perturbations J. Chromatogr. A 1430 (2015) 97-112, http://dx.doi.org/10.1016 j.chroma.2015.07.008.

[43] H.L. Rost, T. Sachsenberg, S. Aiche, C. Bielow, H. Weisser, F. Aicheler S. Andreotti, H.-C. Ehrlich, P. Gutenbrunner, E. Kenar, X. Liang, S. Nahnsen, L. Nilse, J. Pfeuffer, G. Rosenberger, M. Rurik, U. Schmitt, J. Veit, M. Walzer, D. Wojnar, W.E. Wolski, O. Schilling, J.S. Choudhary, L. Malmstrom, R. Aebersold, K. Reinert, O. Kohlbacher, OpenMS: a flexible open-source software platform for mass spectrometry data analysis, Nat. Methods 13 (2016) 741-748, http://dx.doi.org/10.1038/nmeth.3959.

[44] M.R. Berthold, N. Cebron, F. Dill, T.R. Gabriel, T. Kötter, T. Meinl, P. Ohl, K. Thiel B. Wiswedel, KNIME - the konstanz information miner, SIGKDD Explor 11 (2009) 26-31, http://dx.doi.org/10.1145/1656274.1656280.

[45] E. Kenar, H. Franken, S. Forcisi, K. Wörmann, H.-U. Häring, R. Lehmann, P. Schmitt-Kopplin, A. Zell, O. Kohlbacher, Automated label-free quantification of metabolites from liquid chromatography-mass spectrometry data, Mol. Cell Proteom. 13 (2014) 348-359, http://dx.doi.org/10.1074/mcp.M113.031278.

[46] J.D. Storey, A direct approach to false discovery rates, J. R. Stat. Soc. Ser. B Stat Methodol. 64 (2002) 479-498, http://dx.doi.org/10.1111/1467-9868.00346.

[47] P. Van Renterghem, P. Van Eenoo, H. Geyer, W. Schänzer, F.T. Delbeke, Reference ranges for urinary concentrations and ratios of endogenous steroids, which can be used as markers for steroid misuse, in a Caucasian population of athletes, Steroids 75 (2010) 154-163, http://dx.doi.org/10.1016/ j.steroids.2009.11.008.

[48] F. Badoud, E. Grata, J. Boccard, D. Guillarme, J.L. Veuthey, S. Rudaz, M. Saugy, Quantification of glucuronidated and sulfated steroids in human urine by ultra-high pressure liquid chromatography quadrupole time-of-flight mass spectrometry, Anal. Bioanal. Chem. 400 (2011) 503-516, http://dx.doi.org/ 10.1007/s00216-011-4779-8.

[49] A. Kotronoulas, A. Gomez-Gomez, J. Segura, R. Ventura, J. Joglar, O.J. Pozo Evaluation of two glucuronides resistant to enzymatic hydrolysis as markers of testosterone oral administration, J. Steroid Biochem. Mol. Biol. 165 (2017) 212-218, http://dx.doi.org/10.1016/j.jsbmb.2016.06.006.

[50] J.P. Antignac, A. Brosseaud, I. Gaudin-Hirret, F. André, B.L. Bizec, Analytical strategies for the direct mass spectrometric analysis of steroid and corticosteroid phase II metabolites, Steroids 70 (2005) 205-216, http://dx.doi.org 10.1016/j.steroids.2004.11.009.

[51] O.J. Pozo, P. Van Eenoo, W. Van Thuyne, K. Deventer, F.T. Delbeke, Direct quantification of steroid glucuronides in human urine by liquid chromatography-electrospray tandem mass spectrometry, J. Chromatogr. A 1183 (2008) 108-118, http://dx.doi.org/10.1016/j.chroma.2008.01.045.

[52] Competitive Fragmentation Modeling for Metabolite Identification, CFM-ID, 2017. http://cfmid.wishartlab.com (Accessed 18 August 2016).

[53] L. Kornel, Z. Saito, Studies on steroid conjugates-VIII: isolation and characterization of glucuronide-conjugated metabolites of cortisol in human urine J. Steroid Biochem. 6 (1975) 1267-1284, http://dx.doi.org/10.1016/00224731(75)90118-1.

[54] J. Marcos, N. Renau, G. Casals, J. Segura, R. Ventura, O.J. Pozo, Investigation of endogenous corticosteroids profiles in human urine based on liquid chromatography tandem mass spectrometry, Anal. Chim. Acta 812 (2014) 92-104, http://dx.doi.org/10.1016/j.aca.2013.12.030.

[55] W.K. Jerjes, A.J. Cleare, T.J. Peters, N.F. Taylor, Circadian rhythm of urinary steroid metabolites, Ann. Clin. Biochem. 43 (2006) 287-294, http://dx.doi.org/ $10.1258 / 000456306777695564$. 